\title{
Development of Automated BCI System to Assist the Physically Challenged Person Through Audio Announcement With Help of EEG Signal
}

\author{
${ }^{1}$ RAJASHEKHAR U, ${ }^{2}$ NEELAPPA \\ ${ }^{1}$ Dept. of Electronics \& Communication Engineering, Government Engineering College, \\ Kushalnagar, Karnataka, INDIA-571234 and affiliated to Visvesvaraya Technological University, Belagavi, \\ Karnataka, INDIA \\ ${ }^{2}$ Dept. of Electronics \& Communication Engineering, Government Engineering College, Kushalnagar - 571234 \\ and affiliated to Visvesvaraya Technological University, Belagavi, Karnataka, INDIA
}

\begin{abstract}
Individuals face numerous challenges with many disorders, particularly when multiple disfunctions are diagnosed and especially for visually effected wheelchair users. This scenario, in reality creates in a degree of incapacity on the part of the wheelchair user in terms of performing simple activities. Based on their specific medical needs confined patients are treated in a modified method. Independent navigation is secured for individuals with vision and motor disabilities. There is a necessity for communication which justifies the use of virtual reality (VR) in this navigation situation. For the effective integration of locomotion besides, it must be under natural guidance. Electroencephalography (EEG), which uses random brain impulses, has made significant progress in the field of health. The custom of an automated audio announcement system modified to have the help of Virtual Reality (VR) and EEG for training of locomotion and individualised interaction of wheelchair users with visual disability is demonstrated in this study through an experiment. Enabling the patients who were otherwise deemed incapacitated to participate in social activities, as the aim was to have efficient connections. The natural control, feedback, stimuli, and protection these subsequent principles founded this project. Via properly conducted experiments, a multilayer computer rehabilitation system was created that integrated natural interaction assisted by EEG, which enabled the movements in the virtual environment and real wheelchair. For blind wheelchair operator patients this study involved of expounding the proper methodology. For educating the value of life and independence of blind wheelchair users, outcomes proven that VR with EEG signals has that potential. To protect their life straightaway and to report all these disputes, the military system should have high speed, more precise portable prototype device for nursing the soldier health, recognition of solider location and report about health sharing system to the concerned system. FPGA-based soldier's health observing and position gratitude system is proposed in this paper. Reliant on heart rate which is centred on EEG signals the soldier health is observed in systematic bases. By emerging Verilog HDL programming language and executing on Artix-7 development FPGA board of part name XC7ACSG100t the whole work is approved in a Vivado Design Suite. Classification of different abnormalities, and cloud storage of EEG along with type of abnormalities, artifact elimination, abnormalities identification based on feature extraction, exist in the segment of suggested architecture. Irregularity circumstances are noticed through developed prototype system and alert the physically challenged (PHC) individual via audio announcement. An actual method for eradicating motion artefacts from EEG signals that have anomalies in the PHC person's brain has been established, and the established system is a portable device that can deliver differences in brain signal variation intensity. Primarily the EEG signals can be taken and the undesirable artifact can be detached, later structures can be mined by DWT these are the two stages through which artifact deletion can be completed. The anomalies in signal can be noticed and recognized by using machine learning algorithms known as Multirate SVM classifiers, when the features have been extracted using a combination of HMM and GMM. Intended for capable declaration about action taken by a blind person, these result signals are protected in storage devices and conveyed to the controller. Pretending daily motion schedules allows the pretentious EEG signals to be caught. Aimed at the validation of planned system, the database can be used and continued with numerous recorded signals of EEG. The projected strategy executes better in terms of re-storing theta, delta, alpha, and beta (TDAB) complexes of the original EEG with less alteration and a higher signal to noise ratio (SNR) value of the EEG signal which illustrates in the quantitative analysis. The projected method used Verilog HDL and MATLAB software for both formation and authorization of results in order to yield improved results. Since from the achieved results, it is initiated that $32 \%$ enhancement in SNR, $14 \%$ in MSE and $65 \%$ enhancement in recognition of anomalies, hence design is effectively certified and proved for standard EEG signals datasets on FPGA.
\end{abstract}

Key-Words: - EEG, DWT, HMM, GMM and FPGA

Received: January 2, 2021. Revised: April 4, 2021. Accepted: May 7, 2021. Published: May 26, 2021. 


\section{Introduction}

On the way to detect the motion of human heart, screen for cardiovascular diseases, and estimate cardiac and cardiovascular functions, the Electroencephalogram (EEG) signals can be used clinically and these are the primary natal signals. Mobile EEG monitoring systems have emerging to be extensively used to monitor natal signals utmost current days. Due to motion artefact noise, EEG signals are distracting during signal collection. Breathing and muscle contraction as well as unbalanced contact between the skin and the surface of the electrodes. The motion artifact is unexpected noise signals, can make harmful effects in EEG signals, and for analysis of the heart conditions it is not favourable [1]. Towards estimating the perfect EEG signal for monitoring and analysis of heart health, therefore the abolition of motion artifact from EEG signals is essential. Intended for deletion of motion artifact in EEG signals, several authors has projected different approaches. Adaptive filtering (AF), various wavelet transforms (WT), and empirical mode decomposition (EMD) are some of the techniques pre-owned [16]. Using a filter to process the signal is essential for the most sensible way of eliminating motion artefacts $[2,16]$. Adopting the deterministic purpose as a guideline, the filters will remove unnecessary signals without affecting the original signal. Owned to require for precise reference signals as a requirement However, it is incomplete to eliminate EEG motion artifacts. One of the most frequently used de-noising methods in signal processing applications is the Discrete Wavelet transform (DWT) [3-5]. The selection of suitable wavelet functions and thresholds, which makes wavelet method non-adaptive through requires the wavelet transform process. Huang projected an empirical mode decomposition (EMD) process, which is an adaptive technique for decomposing signals into a restricted quantity of intrinsic mode functions (IMFs), towards to resolve the shortcomings of wavelet transform exclusive of flexibility [6]. To eliminate high-frequency noise and baseline wander, Blanco-Velasco suggested an EEG enhancement approach based on the EMD. Frequent difficulty in the EMD process which causes IMFs extracted imperfectly, on the other hand is a mode mixing. Intended for quality removal in wavelet matrices, discrete wavelet transforms have been recommended [7]. Fault analysis, EEG de-noising, disease recognition detection, and transmission line short-circuit fault detection have all used the discrete wavelet transform. The recognition and elimination of Movement Artifacts in EEG Signals using DWT [8]. When a filter is used to minimise power-line intrusion and accurate baseline drift, the RTP and RTCP methods execute superior than the EMD method [9]. Based on discrete wavelet transform and wavelet thresholding, we recommend a process here in this editorial to eradicate the motion artifact from the practical EEG signals [10]. On the way to validate the viability of the projected algorithm, at this juncture the real time EEG were recorded and replicated signals are worn [15]. The projected technique shows enhanced grades in provisions of restorage of the innovative EEG TDAB complexes and enhancement of the signal to noise ratio of the EEG signals related through the Discrete Wavelet Transform and the EMD motion artifact exclusion access [14]. On the way to progress artifact exclusion practice proficient of detecting cardiac interference over a diversity of situation and frame in EEG recording is intend of current exertion. Supplementary description meant for enhanced recognition and organize cardiac associated artifacts, together with potential pulse-related interferences is specified in the projected technique [11-13].

Related Work: The fundamental aim of the Braincomputer interface is to help individuals with disabilities with their treatment and with the help of expression or emotions, the brain-computer interface also assists in operating and managing computeraided systems [17]. To carry out BCI involves detection, analysis, and classification of different types of motor imagery movements to implement real-time control and communication. For the application of $\mathrm{BCI}$, the EEG is commonly employed and is used as a non-invasive system [21]. Classification of limb motor imagery [22], constant movements of the arm, forward hand movement prediction, and backward hand movement estimation [25], P300 suggested potential based character detection, and so on are some of the EEG classes which are based on brain-computer-interface. The identification of left-hand movements and right-hand movements are considered as the fundamental classes of brain-computer-interface [27]. Several techniques are introduced in the proposed literature for categorizing the movements of the arm [28]. Short- 
time Fourier transform (STFT), wavelet transform (WT), and so on are some of the conventional timefrequency techniques. Therefore, Fourier transform is considered as the principal strategy for these techniques and as per the Heisenberg uncertainty principle [25], good time-frequency resolution is not achieved by these approaches. Nowadays, the most well-known technique used is Hilbert Huang Transform (HHT). HHT is appropriate for examining non-stationary signals as well as nonlinear signals. With the help of empirical mode decomposition, the decomposition of an actual signal into a set of intrinsic mode functions is achieved [26] and further Hilbert Huang Transform is applied for every IMF. At last, the classification of features is carried out by approximating the equivalent marginal spectrum and energy spectrum. In the signal processing fields namely seismic signals, biomedical signals, and radar detection [27] this technique is commonly used. The characteristics of the signal and its working are determined by the nonlinear characteristics of the physiological system. Nowadays, some of the research work employs analysis of non-stationary linear discriminant that is reliant on Kalman smoother algorithm on to compute non-stationary constraints and some research work employs algorithms to compute nonlinear dynamic signal constraints namely Coriolis entropy, correlation dimension, Lyapunov exponent [20]. But these constraints are difficult to employ in real-time applications. Thus, these parameters are hard to apply in practice. Therefore, the above drawback can be overcome by the technique introduced by Pincus in 1991 [29]. To determine the signal characteristics as well as to approximate random signal, the only short data point is required by approximate entropy. Approximate entropy possesses better antiinterference ability and anti-noise capacity. Approximate entropy is more useful in the analysis of EEG signals [30] since they consist of both frequency components as well as large random signal components. The computation of autoregressive coefficient and approximate entropy is compared with the other techniques in the previous research works.

2. Proposed Methodology for BCI system to assist the physically challenged person through audio announcement with help of EEG signal

The projected scheme abide of a three-stage technique that assemble raw EEG signals, transform them for artefact removal, extracts features, and detects anomalies. Second, classifiers are used to classify the data, and the results are then interfaced with the FPGA. The procedure is defined in the following section.

\subsection{Methods for EEG signal DENOISING}

Designed for accessing signals which can defend the unique quality waveform and exclusive of distorting the original raw signals, the EEG signal denoising algorithms enhance signal-to noise ratio (SNR) and SSIM are depicted in Fig. 3 (a \& b) . Intended for EEG signal denoising and identical has finished virtually however still should enlarge a scheme which that can recover all the parameters accessible in EEG raw signal, diverse technique have been projected by several composers. We consign a few denoising methods based on median filter, discrete cosine transform, discrete wavelet transform, and the projected technique in the subsequent division [18].

\subsection{About EEG Databases}

The Simulated database and real time database are the two techniques for removing raw EEG waves. For replication dedications, the standard database comprises a selection of EEG signal proceedings of comparable length, which is available with dataset 'em' from the MIT-BIH Noise Stress test Database. In the couple regular and abnormal EEG beats, this distinctive signal comprises time shifting $\mathrm{TDAB}$ morphology. Through accumulating motion artifacts to the EEG dataset signals at diverse levels of Signal to noise ratio, the thunderous EEG datasets were achieved. EEG signals are thought to be the safest way to identify cardiac defects in a broad and realistic sense [19]. The EEG signal acquirement system are commonly big in size and maintain high-accuracy and long-term observing in hospitals. Single-lead EEG recording devices to the 12-lead EEG recording devices are all accessible. Certain wearable healthmonitoring devices conversely, permit for real-time constant observing of a patient's situation over the practice of numerous sensors. The collection of sensor forms, the position of sensors to point, the quantity of sensors, and the hardware desired for data attainment, storage, and broadcast are all portion of the dataset gathering phase in the lifecycle of EEG observing schemes.

\section{PRE-Processing}

Towards improving the precision of expectation and it progresses the excellence of the raw signal by eliminating noise, Powerline interference, baseline wander, electrode touch noise, electrode motion artefacts, and muscle contractions are all examples of noise. Wavelet Transform based, curvelet transform based, and adaptive optical filters are the three best general pre-processing and noise decline methods. The length of the complex, which is similar to the 
cardiac cycle, can be treated better using linear filters, which can avoid phase distortion from fluctuating wave properties. Conversely, averaging practices to the time associated heartbeats are frequently desired to decrease the effect of noise produced by muscle action. Since for controlling the EEG raw signals, the current standard filtering practices has absence of effectiveness. Pre-processing has been used to reserve valuable signal material and familiarize to the patient's characteristics, despite the fact that it is a difficult task is showing in Fig.1.

\subsection{Artifact Noise Extraction}

The documentation of the TDAB wave and or beat recognition in the EEG signal is the most critical procedure in the observing scheme for the recognition of arrhythmia. The glassy of noise and artefacts produced through everyday actions make beat recognition more difficult for ambulatory observing. A patient's EEG signal-to-noise-ratio (SNR) can suffer as a result of engaging in several high-intensity spiritual happenings. Numerous kinds of noise can arise concurrently in the ambulatory EEG. Laterally by these, electrode motion (EM) muscle artefact (MA) and the base line wander (BLW), artefact all of which have a frequency range beyond the EEG signal's frequency limit may have a related morphology to the EEG signal and may alter the medical structures of the signal, which are critical in identifying dissimilar EEG arrhythmias. As related to a noiseless EEG signal, artefacts can disturb the amplitude and frequency. Inadequate electrode-skin interface subsidizes to base line wander and rapid sense. Based on the subject movements, properties of electrode and skin impedance numerous features of the base line wander generosities are contingent. The frequency of the BLW is usually less than one $\mathrm{Hz}$, but it can rise in EEG recordings as the rate of breathing increases due to suitable operative happenings. $20 \mathrm{hz}$ to $1000 \mathrm{hz}$ is the frequency range of electrode motion noise that reduces disturbance without affecting any pathological changes of EEG signal as well as another EM objects results in variation of impedance that is produced by the EM and possess identical components of frequency ranging between 1 to $15 \mathrm{hz}$. This frequency causes signal noise and results in incorrect TDBA system leading to misdiagnoses of atrial fibrillation. The recognition method in attaining perfect and reliable heartbeat measurement for EEG inspecting scheme can be hampered by noise while recording EEG signal. The output of the TDAB indicator must be estimated using the similar test signal database for evaluation. There are no unique noise types or intensity levels that still disturb TDAB morphology or beat recognition proficiency. As a result, in ambulatory cardiac observing, the impact of noise and intensity levels on heartbeat recognition is being estimated, as well as the connection among beat detection and noise features, exclusively artefacts that can morphologically mislead the EEG signal is showing in Fig.1.

\subsection{EEG de-noising with Median Filter (MF)}

The median of the models in a window ' $\mathrm{w}$ ' substitutes the model in the intermediate of the window in median filtering (MF), which is a nonlinear signal smoothing process. Within a window of length $\mathrm{L}$, the defined sequence of $\mathrm{n}$ samples,

$W=\left\{\mathrm{x}_{\mathrm{n}}\right\},-L \leq i \leq L, M=$ median $\left(\mathrm{x}_{\mathrm{n}}\right)$ where $M$ is the median value. For impulse noise dominance, the MF-based denoising method is frequently utilised. The phases for EEG denoising built on MF are as surveys: To filter TDAB complexes and $\mathrm{P}$ waves from the noisy EEG signal, a median filter with a width of $200 \mathrm{~ms}$ is utilised. T waves are detached from the subsequent signal using a 600-ms MF. The baseline of the EEG signal is restricted in the signal from the second filter action, which is detracted from the noisy EEG signal to attain the modified baseline EEG signal.

\section{Feature Extraction using HMM and GMM}

We derived a set of structures expressive of the applicants' perceptive and physical situation in every time occurrence based on outcomes from the neuroscientific literature. The brain's behaviour is defined by rhythmic outlines that span several frequency bands, the concept of which differs marginally amongst readings. Delta $(0.5-4 \mathrm{~Hz})$, theta (4-7 Hz), alpha $1(7-10 \mathrm{~Hz})$, alpha $2(10-13 \mathrm{~Hz})$, beta $(13-30 \mathrm{~Hz})$, and gamma $(30-60 \mathrm{~Hz})$ were the six bands through which EEG is evaluated. Theta and alpha 1 (i.e., lower alpha) frequencies replicate reaction embarrassment and consideration] load like phasic alertness [17] whereas beta movement is related with psychological and physical pressure. Task output in terms of tempo, relevance, and complexity is linked to alpha 2 (i.e., higher alpha) [18]. Multimodal processing or object representation [19] for instance in which gamma ways plays an important role in complex cognitive functions. With a grouping of HMM and GMM, characteristics connected to signal strength and complication were obtained. We calculated the Relative Intensity Ratio as quantify of relative spectral power in each of the six frequency bands for each of the 14 EEG channels [20].We calculated the incident-linked (de)synchronization (ERD/ERS) directory, a wellrecognized quantify of band power change in EEG first proposed by Pfurtscheller and Aranibar [34], 
after separating the power band quality from the EEG signals. It has been identified,

$$
\frac{E R D}{E R S} \%=\frac{\text { Baseline IBP }- \text { Test IBP }}{\text { Base line IBP }} \times 100
$$

where IBP stands for interval band power

One of the most crucial steps in the raw EEG signal monitoring process is feature extraction. It makes a major contribution to cardiac disease diagnosis because it extracts the most critical collection of features from pre-processed raw EEG signals, allowing for improved heartbeat detection. A selection of summarised signal instruction is used to classify patterns among the characteristics. In crisis alignment, these character are obtained using different computation algorithms and techniques. The peak amplitude, heart rate frequency, area under the curve, and time delay between peaks and valleys are all characteristics of EEG signals. Wavelet Transform-based feature extraction, autocorrelation function-based feature extraction (periodic information of EEG signals), principal component analysis-based feature extraction, and normal feature extraction using Fast Fourier Transform are the most frequent characteristic removal method. Time-based, frequency-based using FFT, power spectral density, Gaussian and moving average low-pass filters, and finally a mix of together time and frequency, such as Wavelet Transform, frequency TDAB power, maximum and minimum amplitudes used for heartbeat recognition, are examples of quality removal methods. Discovering the right set of character to confine the true nature of EEG signals is still a challenge. The Discrete Wavelet Transform move towards to enhance the identification of atrial fibrillation by classifying heartbeat abnormalities from EEG signals using time domain, frequency domain, and propagation description.

\subsection{DCT transform}

The discrete cosine transform (DCT) renovates a time domain function into a frequency domain function and is widely used in signal transmission and digital image processing presentations. It is diligently associated to the DFT as the DCT is the actual portion of the DFT, and the 1-D discrete cosine transform of a signal $x(k)$, is the signal $y(k)$ of the same size as $x(k)$, well-defined by:

$y(k)=w(k) \sum_{n=1}^{N} \mathrm{x}(\mathrm{n}) \cos \left(\frac{\pi(2 n-1)(-1)}{2 N}\right)$

Where $\mathrm{N}$ is the length of the noisy EEG signal and $w(k)$ is the DCT quantities estimated by

$w(k)=\left\{\begin{array}{cc}\frac{1}{\sqrt{N}} \quad \text { if } k=1 \\ \sqrt{\frac{2}{N}} \quad \text { if } 2 \leq k \leq N\end{array}\right.$
In order to find their inverse form, just by switch $k$ and $n$ in y (k) equation. On behalf of extremely interrelated records, the DCT offers admirable energy density. We observed that the DCT spectrum is positioned at the low frequency, which resembles to the first quantities of the DCT transform, after the energy compaction property of DCT was conversed and offered with detail to some regular EEG signals proceedings. We hypothesise that the BW artefact in the EEG signal corresponds to the first coefficients obtained using a one-dimensional DCT transform.

\section{Classification}

Planning exact classifier created on Neural Networks with SVM classifiers can be used to categorize data into regular and irregular classifications. Machine knowledge, a form of artificial intelligence (AI) that provides computers the capability to study individually with trained programs, has been used to train the system with altered data sets and it can now recognize the records individually. Exhausting the data to notice potential designs in data and alter program actions consequently is the method of machine learning. Unsupervised and supervised learning are two types of machine learning techniques. In directed education, preparation data proceeds the form $(\mathrm{Xn}, \mathrm{Yn}) \mathrm{n}=1,2, \ldots \mathrm{N}$, where $\mathrm{Xn}$ are instances from an input space $X$ and $Y n$ are produced by an indefinite unplanned process with $\mathrm{Xn}$ as input. Classification and regression are two of the best public difficulties in administered education. Unverified Learning, on the otherside, is given a set of samples $\mathrm{Xn}, \mathrm{n}=1,2, \ldots . \mathrm{N}$, but the labels Yn are not manageable. The accessible results are discussed to as unlabeled data since $\mathrm{Yn}$ is unattainable. In this instance, pattern categorizations and structure appreciation are generally associated to the learning complications, such as the number of data clusters, the centre of clusters, and the clusters themselves of the input data. We castoff the Support Vector Machine (SVM) as a machine learning algorithm which can afford improved outcomes in splitting datasets and has more robust presentation when trade with data improbability in our suggested method. The datasets were first composed and deposited in dissimilar training and testing sets that were dissimilar from each other, and then the SVM algorithm was enforced to the preparing data sets using VLSI programming. 
NeuroSky Mind ware Mobile for EEG Signal

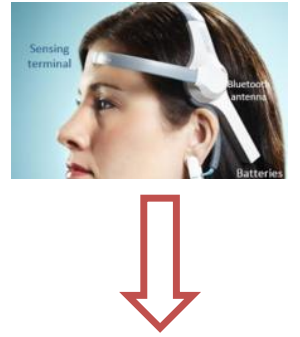

Pre-processed Signal at $10 \mathrm{~Hz}$, $20 \mathrm{~Hz}, 30 \mathrm{~Hz}$ and $40 \mathrm{~Hz}$

$$
\begin{aligned}
& \text { weight for cluster }\left(w_{j}^{i}\right) \\
& =\sum_{l=1}^{m} w_{i l}
\end{aligned}
$$

Formation of Clusters for Classifications

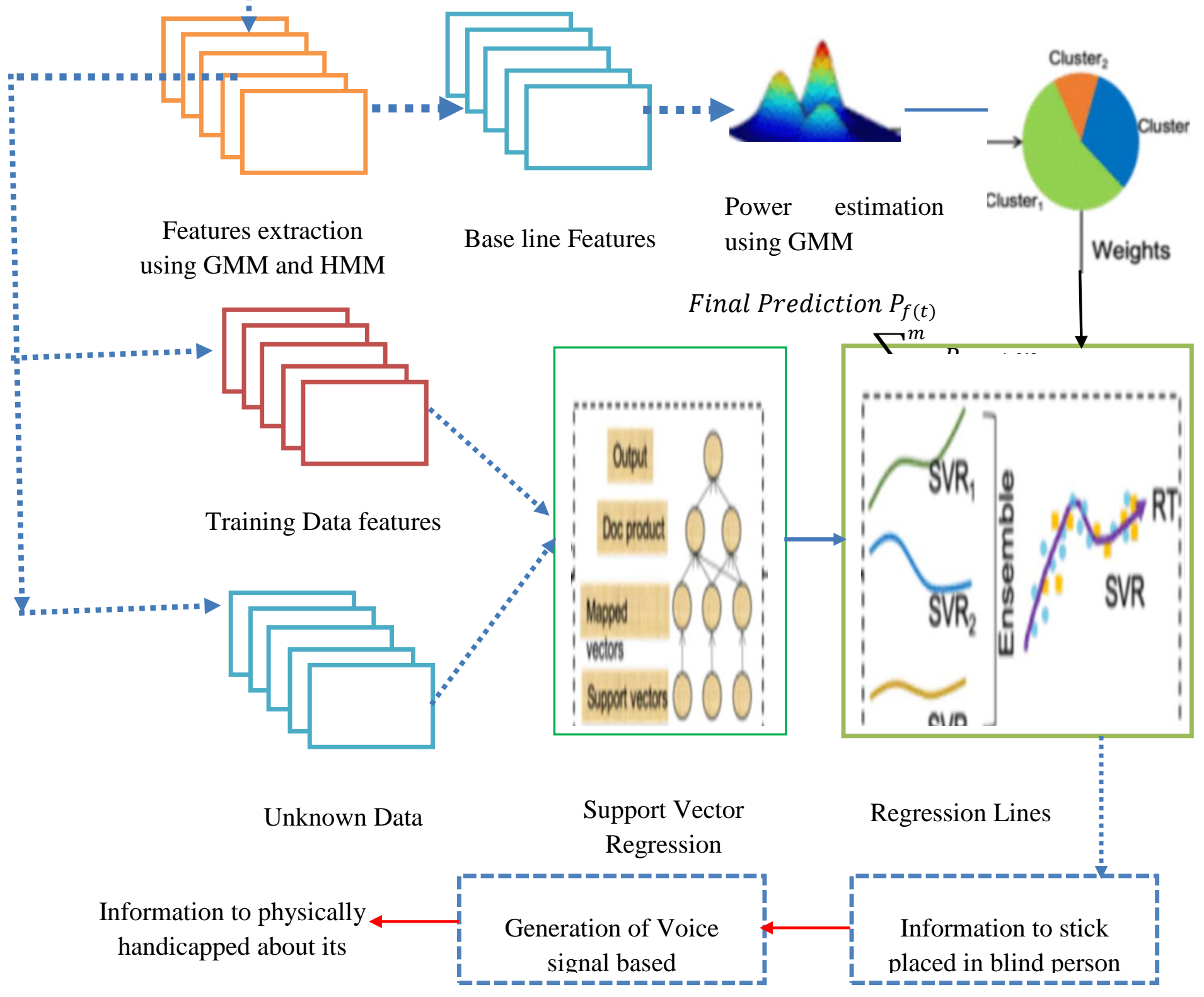

Fig.1 Proposed design flow diagram of abnormalities detection for military people's health monitoring system

The removed structures are related to the training data set structures through grouping. When dealing with unfamiliar data, cross-justification is used to predict how a machine learning algorithm will work. Moderate the errors that come with machine learning algorithms that form a premise and then estimate it using the same data is the key objective. The data is erratically separated into K division in K-fold crossauthentication. One division of the data sets is used for analysis, while the residual data sets are used for 
using the same data is the key objective. The data is erratically separated into $\mathrm{K}$ division in $\mathrm{K}$-fold crossauthentication. One division of the data sets is used for analysis, while the residual data sets are used for training sets, and each division set must be dignified $\mathrm{K}$ times before being estimated. Three-fold crossvalidation is used to test the feasibility of the proposed method. Adopting a classifier, every pleat is castoff to examine the precision. Although the classifiers are mongrelised, the cataloguing exactness derived from them is taken into description as the burden of the identical. The complete proposed design is implemented on Artix-7 FPGA and it is installed in wheelchair. The electrodes are placed on head of the patient to acquire the EEG and the final outputs are controlling the wheelchair and at same time it will announce the directions to blind person.

\subsection{Performance Evaluation}

TDAB recognition, heart rate, SNR, PSNR, precision, and error rate are some of the constraints that can be used to assess the proficiency of the projected procedures are shown in Fig. 4 (a \& b). Classifier Evaluation metrics: To determine the projected technique's grouping efficiency, three key analytical procedures were cast-off. To begin, we measured the algorithm classifications' accuracy as the proportion of all true classifications across all classifications as shown in Fig.2. First, we restrained the algorithm classifications precision as the proportion of all true classifications across all classifications.

$$
\text { Accuracy }=\frac{\Sigma(\mathrm{TP}+\mathrm{TN})}{\Sigma(T P+T N+F P+F N)}
$$

Second, we calculated the false error rate (FER) of algorithm classifications as the proportion of falsely labelled as NCC out of all NCC classifications according to the equation, $\mathrm{FER}=\frac{\Sigma(\mathrm{FN})}{\Sigma(T N+F N)}$ And the third, we calculated the sensitivity's' of the classification algorithm is given by

$\mathrm{s}=\frac{\mathrm{HR}-\mathrm{FAR}}{(1-F A R)} \quad$ Where HR is the hit rate and FAR is the false acceptance rate, can be defined below, $\mathrm{HR}=\frac{\Sigma(\mathrm{TP})}{\Sigma(T P+F N)}$ and $\mathrm{FAR}=\frac{\Sigma(\mathrm{FP})}{\Sigma(F P+T N)}$

When the categorisations are right, the efficiency's' equals 1 and FAR equals 0. Separately, precision, FAR, and s were estimated.

\section{Results and Discussion}

To identify precisely all TDAB intermissions deprived of embracing the wrong documentation for altered level of noises investigate on detecting anomalies in EEG signals in the field of VLSI is capable of recommended. Noises like muscle artifacts, electrode signal and baseline warder which are frequently fulfill sideways with MIT-BIH standard databases regardless of the dissimilar passions. Error recognition identification of TDAB internals and faults is exaggerated by both of these evasion noises. Each TDAB intermission is divided into three categories: false positive (FP), true positive (TP), and false negative (FN) to confirm the heart rate measurements (FN). FN is contingent on nonidentified peaks in TDAB intermissions, $F P$ is signifies the number of non-TDAB or noisy TDAB identified peaks and TP is contingent on TDAB complex and is number of TDAB peaks identified. Enactment metrics such as sensitivity, accuracy, false error rate, and predictively positive are evaluated centred on these constraints. On the source of 73 recorded databases from the MIT-BIH arrhythmia centre, the recognition of heart rate on a consistent EEG signal is examined. All of the reported datasets are associated with various anomalies, as described below, Table 1. Each dataset comprises 3600 samples, which are rehabilitated to binary using MATLAB source code and stored in the FPGA's Block-RAM (BRAM). When compared to previous works, all projected practices for precise irregularity recognition can generate performance with a 99 percent exactness and there is development of $5 \%$.

Table 1 Database record count with type of abnormalities.

\begin{tabular}{|l|l|l|}
\hline $\begin{array}{l}\text { Database } \\
\text { record }\end{array}$ & Name of the abnormalities & $\begin{array}{l}\text { Database } \\
\text { count }\end{array}$ \\
\hline $1-12$ & Activity:1(Pain in Brain) & 12 \\
\hline $1-18$ & $\begin{array}{l}\text { Activity:2(Heart Rate } \\
\text { variations) }\end{array}$ & 18 \\
\hline $1-16$ & $\begin{array}{l}\text { Activity:3(Based on hands } \\
\text { movement) }\end{array}$ & 16 \\
\hline $1-15$ & $\begin{array}{l}\text { Activity:4(Left Legs } \\
\text { movements) }\end{array}$ & 15 \\
\hline $1-12$ & $\begin{array}{l}\text { Activity:5(Right Legs } \\
\text { movements) }\end{array}$ & 12 \\
\hline
\end{tabular}

The Table 2 shows in positions of anomaly recognition and recognition precision in percentage, the projected effort and present effort are equated. The projected automated abnormality recognition system is originate to be proficient of noticing anomalies from the consistent database, which are from the MIT-BIH arrhythmia database the suggested device can notice Atrial fibrillation with 100 percent accuracy, Atrial flutter with 68.2 percent accuracy, Normal Sinus rhythm with 93 percent accuracy, Sinus Tachycardia with 65 percent 
accuracy, and Ventricular Fibrillation with 100 percent precision, among other diseases are shown in Table 2. The projected scheme has precision of 85.24 percent on average.

Table 2 Comparative analysis of proposed research work with existing work in terms of detection of different abnormalities and accuracy.

\begin{tabular}{|c|c|c|c|c|}
\hline $\begin{array}{l}\text { Databa } \\
\text { se } \\
\text { record } \\
\text { numbe } \\
\text { r }\end{array}$ & $\begin{array}{l}\text { Name of } \\
\text { the } \\
\text { abnormal } \\
\text { ity }\end{array}$ & $\begin{array}{l}\text { Detecte } \\
\mathrm{d} \\
(\mathrm{YES} / \mathrm{N} \\
\mathrm{O})\end{array}$ & $\begin{array}{l}\text { Propos } \\
\text { ed } \\
\text { work } \\
\text { Accura } \\
\text { cy }\end{array}$ & $\begin{array}{l}\text { Existin } \\
\text { g work } \\
\text { Accura } \\
\text { cy }\end{array}$ \\
\hline $1-12$ & $\begin{array}{l}\text { Activity: } \\
1\end{array}$ & Yes & $100 \%$ & $94.2 \%$ \\
\hline $\begin{array}{l}1-2 \\
3 \\
4-5 \\
6 \\
7 \\
8 \\
9 \\
10 \\
11-12 \\
13 \\
14-15 \\
16 \\
17-18 \\
\end{array}$ & $\begin{array}{l}\text { Activity: } \\
2\end{array}$ & $\begin{array}{l}\text { Yes } \\
\text { No } \\
\text { Yes } \\
\text { No } \\
\text { Yes } \\
\text { No } \\
\text { Yes } \\
\text { No } \\
\text { Yes } \\
\text { No } \\
\text { Yes } \\
\text { No } \\
\text { Yes }\end{array}$ & $72 \%$ & $68.2 \%$ \\
\hline $\begin{array}{l}1-12 \\
13 \\
14-16 \\
\end{array}$ & $\begin{array}{l}\text { Activity: } \\
3\end{array}$ & $\begin{array}{l}\text { Yes } \\
\text { No } \\
\text { Yes } \\
\end{array}$ & $93 \%$ & $81 \%$ \\
\hline $\begin{array}{l}1 \\
2-4 \\
5-7 \\
8-13 \\
14-15 \\
\end{array}$ & $\begin{array}{l}\text { Activity: } \\
4\end{array}$ & $\begin{array}{l}\text { No } \\
\text { Yes } \\
\text { No } \\
\text { Yes } \\
\text { No } \\
\end{array}$ & $65 \%$ & $70 \%$ \\
\hline $1-12$ & $\begin{array}{l}\text { Activity: } \\
5\end{array}$ & Yes & $100 \%$ & $89.5 \%$ \\
\hline
\end{tabular}

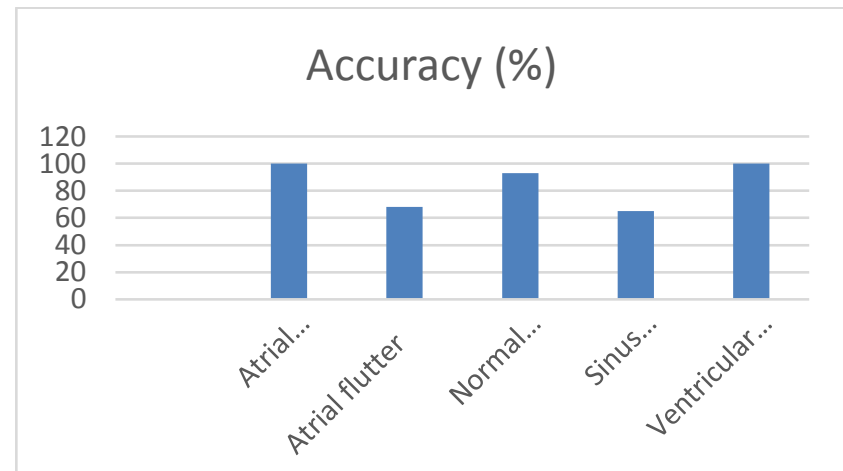

Fig.2. Detection of abnormalities in EEG signals by proposed system in teams of Accuracy

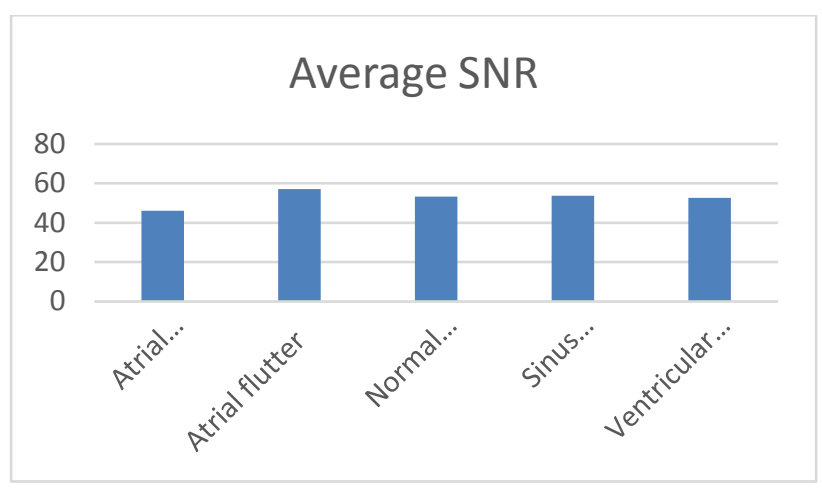

(a)

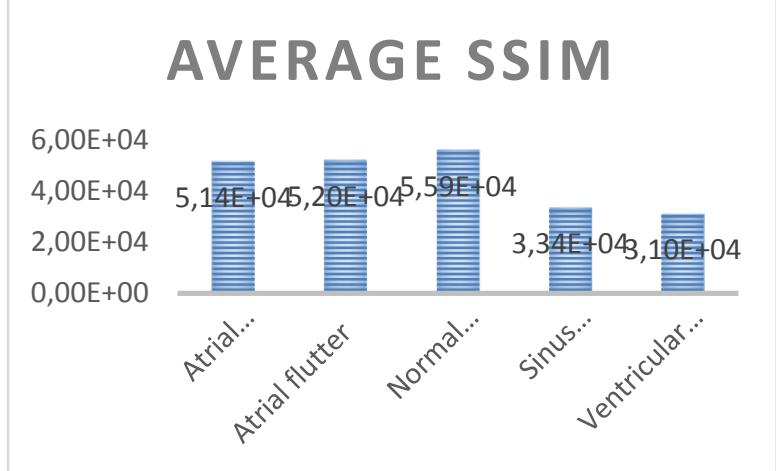

(b)

Fig.3. (a) Average SNR of all types of abnormalities

(b) Average SSIM of all types of abnormalities

Uniqueness abstraction and categorizations methods are worthy sufficient to perceive all TDAB intervals and predictively positive (PP) for all the datasets which are high sensitiveness near the recognition of anomaly of heart pulse observed in advised filters, after positive cataloguing of anomalies into dissimilar classifications.

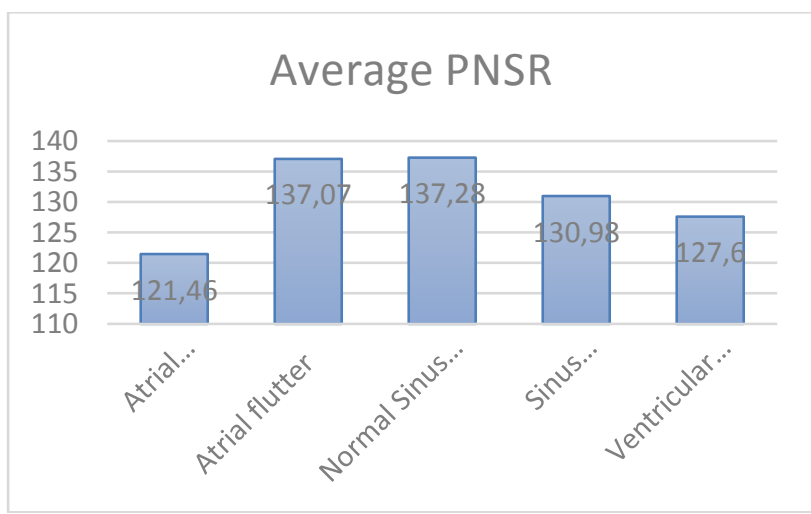

(a) 


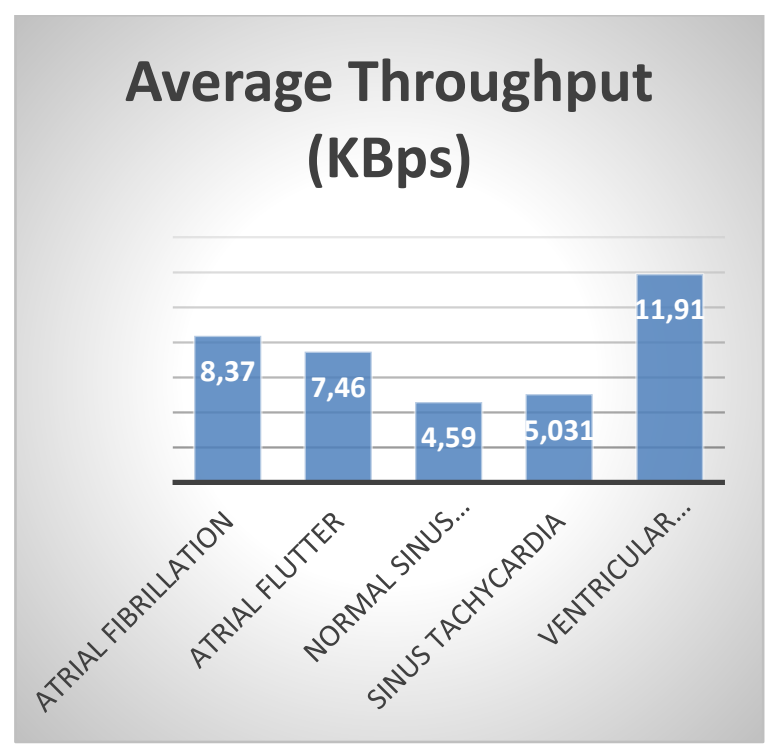

(b)

Fig.4.(a) Average PSNR of all types of abnormalities (b) Average throughput of all types of abnormalities

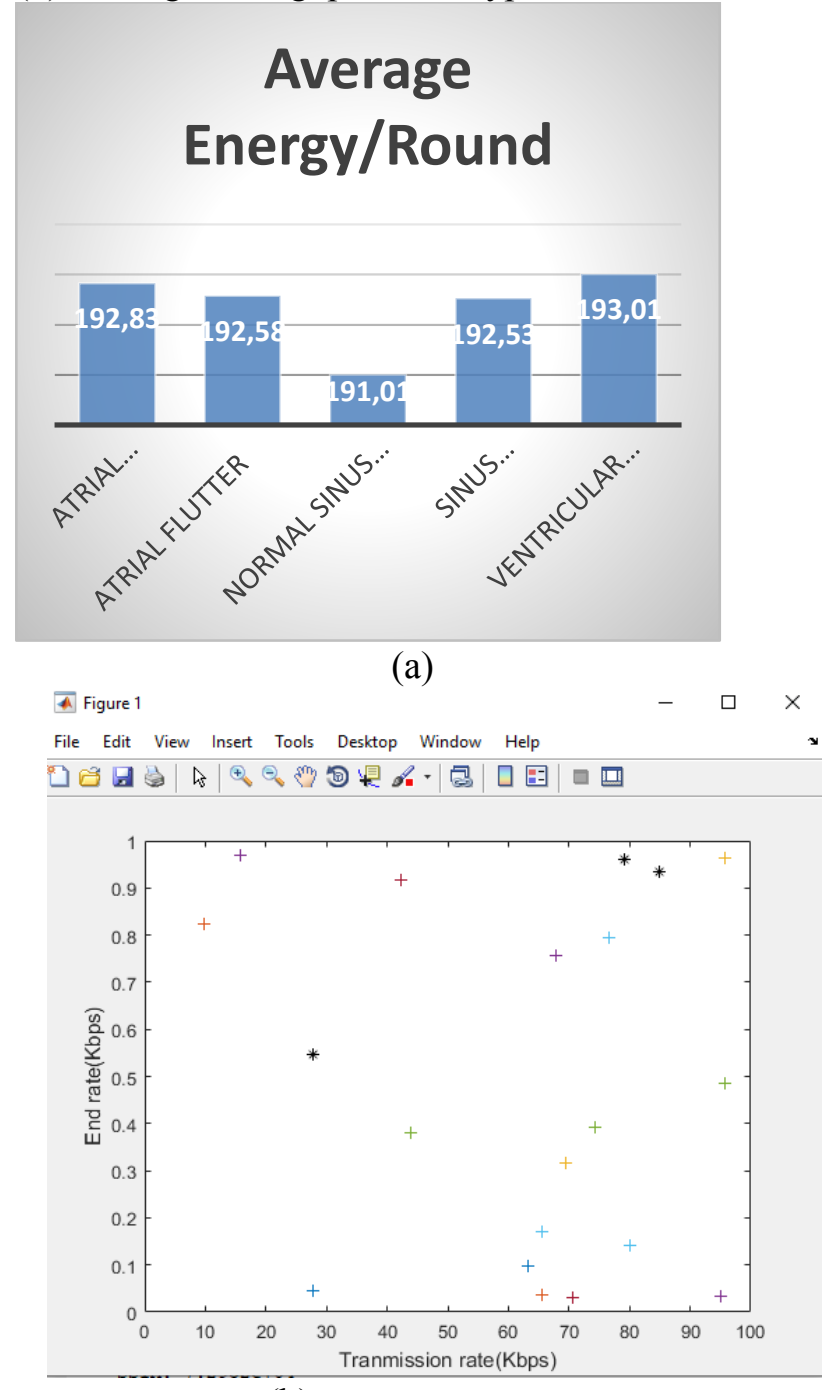

(b)

Fig.5. (a) Average Energy per round of all types of abnormalities (b) Packet transmission into cloud
DCY has excellent energy compression and power indulgence abilities related to STFT, FF,. The Fig. 5(b) shows the EEG database record no.15 of atrial fibrillation and 12 of atrial flutter and their energy compaction results shown in Fig. 6(a\&c). Through lettering Verilog HDL programming language and manufactured by VIVADO design suite 2018.1 automated military soldiers health monitoring scheme is effectively planned and projected. The RTL design diagram is shown in Fig.7, the simulated results are shown in Fig.8. The simulated results of artifacts deletion and types removed results are shown in Fig.9 the manufactured effects of the projected health observing scheme is shown in Table. 4.

Table 3 Performance analysis and comparison of proposed research work with existing works in terms of throughput and energy per round.

\begin{tabular}{|c|c|c|c|c|}
\hline $\begin{array}{l}\text { Databas } \\
\mathrm{e} \\
\text { record } \\
\text { number }\end{array}$ & $\begin{array}{l}\text { Name of } \\
\text { the } \\
\text { abnormalit } \\
y\end{array}$ & $\begin{array}{l}\text { Detected } \\
\text { (YES/N } \\
\text { O) }\end{array}$ & Throughpu & $\begin{array}{l}\text { Energy } \\
\text { per } \\
\text { round }\end{array}$ \\
\hline $\begin{array}{c}1 \\
2 \\
3 \\
4 \\
5 \\
6 \\
7 \\
8 \\
9 \\
10 \\
11 \\
12\end{array}$ & Activity:1 & $\begin{array}{l}\text { YES } \\
\text { YES } \\
\text { YES } \\
\text { YES } \\
\text { YES } \\
\text { YES } \\
\text { YES } \\
\text { YES } \\
\text { YES } \\
\text { YES } \\
\text { YES } \\
\text { YES }\end{array}$ & $\begin{array}{c}98.1 \\
2.8350+03 \\
4 \\
2.8584 \mathrm{e}+0 \\
3 \\
1.3805 \mathrm{e}+0 \\
4 \\
1.9570 \mathrm{e}+0 \\
4 \\
5.9426 \mathrm{e}+0 \\
3 \\
1.3690 \mathrm{e}+0 \\
4 \\
1.3147 \mathrm{e}+0 \\
4 \\
462.2741 \\
1.5519 \mathrm{e}+0 \\
4 \\
6.092 \mathrm{e}+03 \\
916.9429\end{array}$ & $\begin{array}{c}197.204 \\
4 \\
193.877 \\
1 \\
189.313 \\
9 \\
193.280 \\
9 \\
190.079 \\
5 \\
193.280 \\
9 \\
190.079 \\
5 \\
189.237 \\
8 \\
193.092 \\
8 \\
190.274 \\
1 \\
188.374 \\
5 \\
191.293 \\
9\end{array}$ \\
\hline $\begin{array}{l}1 \\
2 \\
3 \\
4 \\
5 \\
6 \\
7 \\
8 \\
9\end{array}$ & Activity:2 & $\begin{array}{c}\text { Yes } \\
\text { Yes } \\
\text { No } \\
\text { YES } \\
\text { YES } \\
\text { No } \\
\text { YES } \\
\text { No } \\
\text { YES }\end{array}$ & $\begin{array}{c}7.2972 \mathrm{e}+0 \\
3 \\
8.6694 \mathrm{e}+0 \\
3 \\
\text { NA } \\
1.4161 \mathrm{e}+0 \\
4 \\
2.6194 \mathrm{e}+0 \\
3\end{array}$ & $\begin{array}{c}194.487 \\
7 \\
192.880 \\
\text { NA } \\
192.499 \\
0 \\
192.495 \\
3 \\
\text { NA }\end{array}$ \\
\hline
\end{tabular}




\begin{tabular}{|c|c|c|c|c|}
\hline 10 & & No & NA & 191.099 \\
\hline 11 & & YES & $5.3785 \mathrm{e}+0$ & 0 \\
\hline 12 & & YES & 3 & NA \\
\hline 13 & & No & NA & 188.167 \\
\hline 14 & & YES & $3.3495 \mathrm{e}+0$ & 7 \\
\hline 15 & & YES & 3 & NA \\
\hline 16 & & No & NA & 192.881 \\
\hline 17 & & YES & $7.1088 \mathrm{e}+0$ & 3 \\
\hline \multirow[t]{12}{*}{18} & & YES & 3 & 194.012 \\
\hline & & & $6.238 \mathrm{e}+03$ & 5 \\
\hline & & & NA & NA \\
\hline & & & $1.0104 \mathrm{e}+0$ & 194.072 \\
\hline & & & 3 & 2 \\
\hline & & & $1.6443 \mathrm{e}+0$ & 193.660 \\
\hline & & & 4 & 6 \\
\hline & & & NA & NA \\
\hline & & & $1.1840 \mathrm{e}+0$ & 192.641 \\
\hline & & & 4 & 9 \\
\hline & & & $5.3875 \mathrm{e}+0$ & 192.098 \\
\hline & & & 3 & 0 \\
\hline 1 & \multirow{31}{*}{ Activity:3 } & YES & $1.6992 \mathrm{e}+0$ & 184.296 \\
\hline 2 & & YES & 4 & 6 \\
\hline 3 & & YES & $1.7338 \mathrm{e}+0$ & 195.162 \\
\hline 4 & & YES & 4 & 8 \\
\hline 5 & & YES & 709.4419 & 189.057 \\
\hline 6 & & YES & 131.6711 & 7 \\
\hline 7 & & YES & $2.9805 \mathrm{e}+0$ & 188.453 \\
\hline 8 & & YES & 3 & 6 \\
\hline 9 & & YES & $1.6783 \mathrm{e}+0$ & 186.627 \\
\hline 10 & & YES & 3 & 4 \\
\hline 11 & & YES & $1.3413 \mathrm{e}+0$ & 188.891 \\
\hline 12 & & YES & 3 & 0 \\
\hline 13 & & No & 565.1844 & 189.576 \\
\hline 14 & & YES & 586.9359 & 8 \\
\hline 15 & & YES & $1.1809 \mathrm{e}+0$ & 194.829 \\
\hline \multirow[t]{16}{*}{16} & & YES & 3 & 8 \\
\hline & & & $1.4731 \mathrm{e}+0$ & 192.353 \\
\hline & & & 4 & 3 \\
\hline & & & $7.181 .5 \mathrm{e}+$ & 191.176 \\
\hline & & & 03 & 2 \\
\hline & & & NA & 188.518 \\
\hline & & & $5.4624 \mathrm{e}+0$ & 7 \\
\hline & & & 3 & 193.234 \\
\hline & & & 154.8631 & 2 \\
\hline & & & 464.5434 & NA \\
\hline & & & & 195.158 \\
\hline & & & & 8 \\
\hline & & & & 193.985 \\
\hline & & & & 6 \\
\hline & & & & 193.855 \\
\hline & & & & 2 \\
\hline 1 & \multirow{4}{*}{ Activity:4 } & No & NA & NA \\
\hline 2 & & Yes & $3.3701 \mathrm{e}+0$ & 194.948 \\
\hline 3 & & Yes & 3 & 6 \\
\hline 4 & & Yes & & \\
\hline
\end{tabular}

\begin{tabular}{|c|c|c|c|c|}
\hline 5 & & No & $5.0912 \mathrm{e}+0$ & 192.789 \\
6 & & No & 3 & 6 \\
7 & & No & $4.2016 \mathrm{e}+0$ & 193.251 \\
8 & & Yes & 3 & 2 \\
9 & & Yes & NA & NA \\
10 & & Yes & NA & NA \\
11 & & Yes & NA & NA \\
12 & & Yes & $5.9014 \mathrm{e}+0$ & 189.212 \\
13 & & Yes & 3 & 6 \\
14 & & No & $5.3201 \mathrm{e}+0$ & 195.480 \\
15 & & No & 3 & 2 \\
& & & $6.1702 \mathrm{e}+0$ & 193.851 \\
& & 3 & 3 \\
& & & $6.6821 \mathrm{e}+0$ & 196.086 \\
& & 3 & 9 \\
& & & $3.3505 \mathrm{e}+0$ & 191.464 \\
& & & 3 & 1 \\
& & & $5.1926 \mathrm{e}+0$ & 185.853 \\
& & & 3 & 7 \\
& & & NA & NA \\
& & & NA & AN \\
\hline & & & & \\
\hline & & & & \\
\hline
\end{tabular}

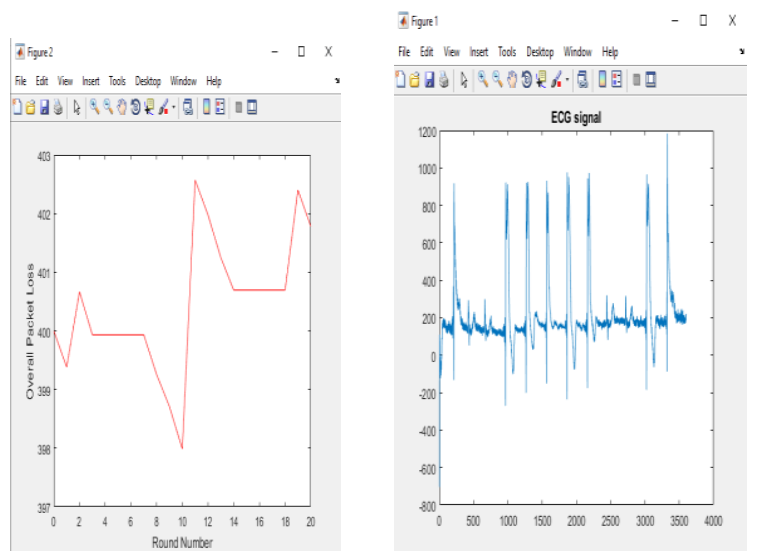

(a)

(b)

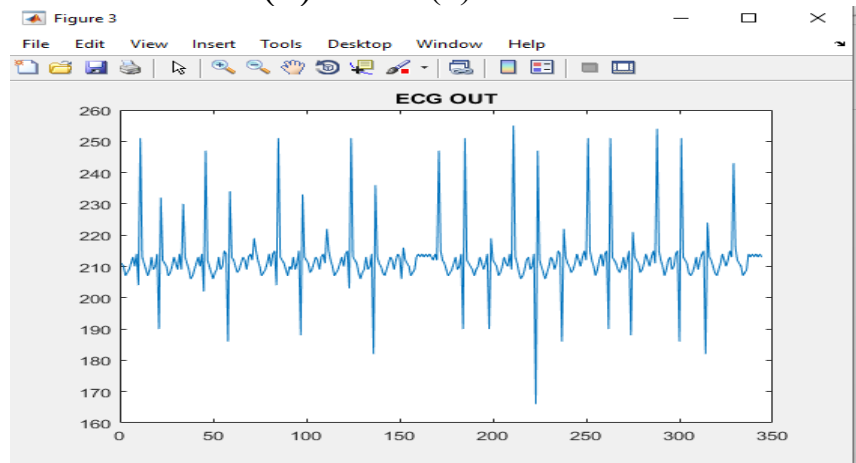

(c)

Fig.6 (b) Database EEG signals, (a) packet loss w.r.t number of round to upload the packet into cloud for storage (c) After removal of artifacts in MATLAB 


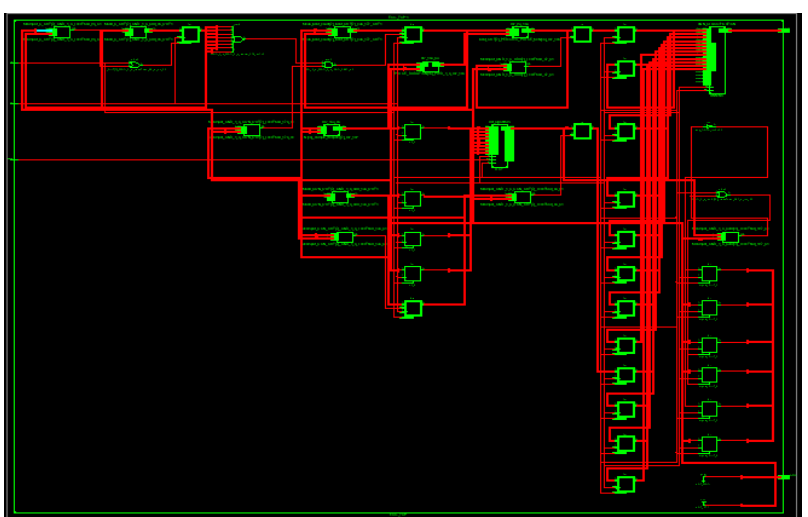

Fig.7. RTL diagram of proposed automated abnormality detection in EEG for monitoring of solider health

显栺

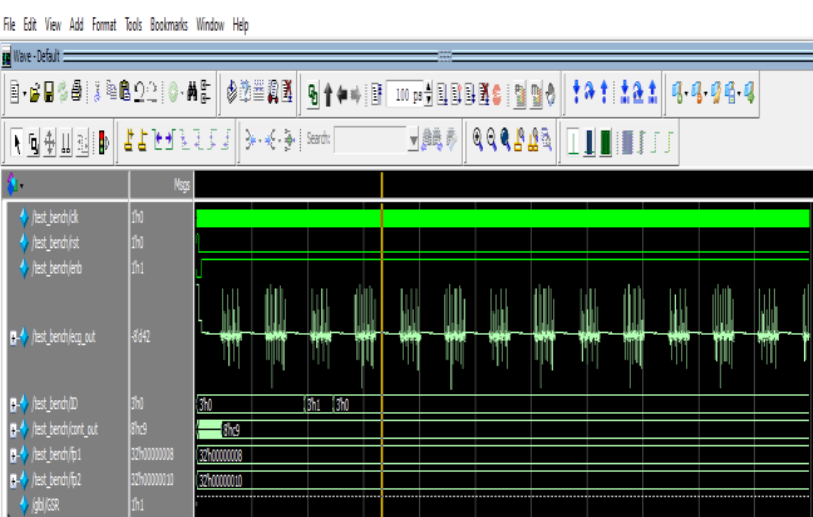

Fig.8. Simulated results of EEG signals for automatic detection of abnormality

Since the device is quick and has been tested against a standard database, the prototype module established will undoubtedly aid in the monitoring of soldiers' health conditions through EEG signals. The outcomes showed that, the system operating at $113.148 \mathrm{MHz}$, power consumption is of $0.082 \mathrm{Watts}$, number of LUT's utilized is 485, area (no of slice register) is 284 and delay is $4.419 \mathrm{~ns}$. The ANN classifier classifies the database into different abnormalities after processing the EEG signals via the established module, and the type of abnormality is sent to concern (base station, where soldiers' health is monitored). Soldier can acquire cure proximately to protect their life therefore over GSM, the message about soldier's health situations is up-to-date to medical professionals or interest. The type of anomaly is communicated via FPGA to Arduino Uno board and interfaced with GSM module to inform the type of anomaly in the custom letter to the concerns after database categorizations. Enabling soliders to attain treatment exact rapidly the erected archetype segment took $2.5 \mathrm{~ns}$ to comprehensive the whole process.

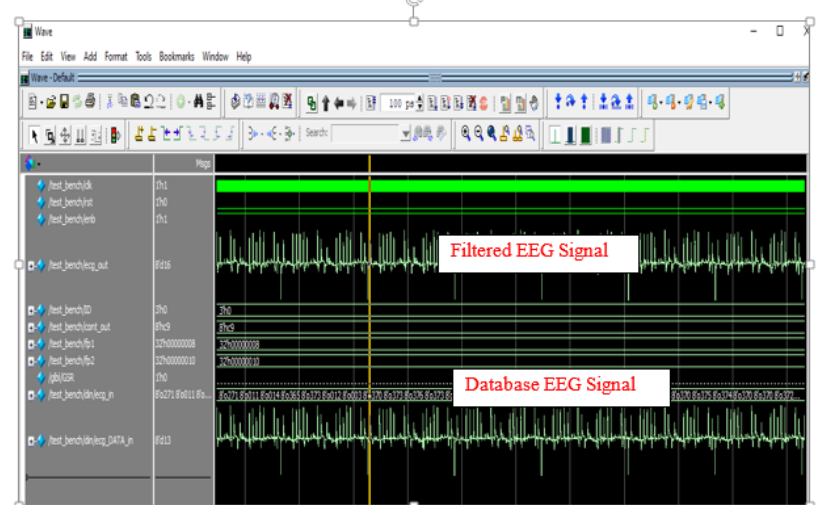

Fig.9. Artifacts removed simulated results of EEG signals for automatic detection of abnormality

For the vast majority of records in the MIT-BIH Arrhythmia Database, all beat detector algorithms performed admirably. Nonetheless, there were a few records in this database with dynamic signals due to abnormal beats and noise effects, such as records 12 , $18,16,15$, and 12 . These documents were previously used to evaluate the noise robustness of previous studies. The signal from record 73 is the most difficult record in the MIT-BIH Arrhythmia Database, according to the PhysioNet web-based resource, due to the signal's prevailing rhythm of irregular beats. Table 1 provides a comparison of algorithm output for a few challenging records, including records $12,18,16,15$, and 12 . Document numbers $12,18,16,15$ and 12 are few examples results revealed that the beat indicator can switch both regular and irregular beat indications. Signal from record 20 showed a normal, atrial late, and premature ventricular contraction beat while the signal from record 73 showed a regular and mixed ventricular beat. The EEG signal for record 53 is a combination of regular and paced ventricular reduction beats.

\section{Conclusion}

Pre-processing is necessary for any EEG signal in order to eliminate the artefact result. For artefact exclusion in raw EEG signals, the advised method employs the median filter, HMM, GMM, and DCT, with the height constants of the DCT renovate zeroed. To classify correct datasets as production, SVM classifiers was utilised. The output signal was then provided to RTP and RTCP before being stored in packing policies. FPG will attach these results. Projected algorithm is more operative and offers improved enactment in terms of both SNR metric and enactment estimation aspects specified by results. To enhance integration in the training of visually diminished wheelchair users, a computational framework including hardware, software, and 
computational methods was established in this

\begin{tabular}{|c|c|c|}
\hline Parameter & $\begin{array}{l}\text { Existing } \\
\text { work }\end{array}$ & $\begin{array}{l}\text { Proposed work for } \\
\text { Automated } \\
\text { abnormality } \\
\text { detection system }\end{array}$ \\
\hline $\begin{array}{l}\text { EEG signal } \\
\text { coefficients bit } \\
\text { size }\end{array}$ & 8 & 8 \\
\hline $\mathrm{FF}$ & 452 & $267(1 \%)$ \\
\hline Delay & $6.42 \mathrm{~ns}$ & $4.419 \mathrm{~ns}$ \\
\hline Area (Slices) & 451 & $284(1 \%)$ \\
\hline $\begin{array}{l}\text { Power } \\
(\mathrm{Vdd}=1.8 \mathrm{~V})\end{array}$ & $1.32 \mathrm{~W}$ & $0.082 \mathrm{~W}$ \\
\hline Slice registers & 280 & 159 \\
\hline AND/OR gates & 2 & 0 \\
\hline No of LUT's & 569 & 485 \\
\hline Throughput & $0.872 \mathrm{Gbp}$ & $\begin{array}{l}\text { Size of the } \\
\text { data/Delay=1.810 } \\
\text { Gbps }\end{array}$ \\
\hline Latency & $61.4 \mathrm{~ns}$ & $\begin{array}{l}\text { Product of Delay } \\
\text { and size of } \\
\text { data }=4.419 \mathrm{~ns} * 8= \\
35.352 \mathrm{~ns}\end{array}$ \\
\hline $\begin{array}{l}\text { No of } \\
\text { computational }\end{array}$ & 670 & 510 \\
\hline $\begin{array}{l}\text { Area-delay } \\
\text { product }\end{array}$ & $84301 \mathrm{~ns}$ & $\begin{array}{l}4.145 * 35.352 \mathrm{~ns} \\
=52380.365 \mathrm{~ns}\end{array}$ \\
\hline $\begin{array}{l}\text { Power and } \\
\text { Delay product }\end{array}$ & $\begin{array}{l}\text { 64013m } \\
\text { W/ns }\end{array}$ & $14.653404 \mathrm{~mW} / \mathrm{ns}$ \\
\hline Frequency & $109 \mathrm{MHz}$ & $113.148 \mathrm{MHz}$ \\
\hline
\end{tabular}

learning.

The patient was able to assess the tool and conduct clinical procedures in the case study that was tested. Over use of the wheelchair and replicate the movement's proficient in the VE the test matter was still able to tread over predefined movements. The use of a wheelchair adapted to have the assistance of VR and EEG for training of locomotion and individualised interaction of wheelchair users with visual disability is illustrated in this study through an experiment. The aim was to have efficient connections, enabling patients who were otherwise deemed incapacitated to participate in social activities. Natural regulation, feedback, stimuli, and protection were the guiding principles for this scheme. Via properly directed experiments, a multilayer computer restoration scheme was established that incorporated natural interface backed by EEG, which permitted the activities in the Virtual Environment and real wheelchair. The aim of this study was to establish a suitable solution for blind wheelchair users. The findings showed that integrating Virtual Reality with EEG signals would help blind wheelchair users improve their eminence of life and individuality.

\section{References}

[1]. M. Zima, P. Tichavsk, K. Paul, andV. Kraj ${ }^{` c a,}$ "Robust removal of short-duration artifacts in long neonatal EEG recordings using waveletenhanced ICA and adaptive combining of tentative reconstructions, Physiological Measurement," vol. 33, no. 8, pp.N39-N49, 2012.

[2]. Al-Qazzaz, N. K., Hamid Bin Mohd Ali, S., Ahmad, S. A., Islam, M. S., and Escudero, J. (2017), "Automatic artifact removal in EEG of normal and demented individuals using ICAWT during working memory tasks. Sensors",doi: 10.3390/s17061326

[3]. Haumann, N. T., Parkkonen, L., Kliuchko, M., Vuust, P., and Brattico, E. (2016), "Comparing the performance of popular MEG/EEG artifact correction methods in an evoked-response study",Comput. Intell. Neurosci. 2016, 1-10. doi: 10.1155/2016/7489108

[4]. S. W. Yoon, S. D. Min, Y. H. Yun, S. Lee and M. Lee, "Adaptivemotion artifacts reduction using 3-axis accelerometer in E-textile EEG measurement system", J. Med. Sys., 32: 101106, 2008.

[5]. B.N. Singh and A.K. Tiwari, "Optimal selection of wavelet basis function applied to EEG signal denoising," Digital Signal Processing, vol. 16, no. 3, pp. 275-287, 2006

[6]. T. Zikovet al. "A wavelet based de-noising technique for ocular artifactcorrection of the electroencephalagram. In Proceedings of the Second Joint EMBS/BMES", Conference, Houston, pages 98-105, 2002

[7]. MJ Vidya and ShruthiSadasiv, " $A$ Comparative Study on Removal of Noise in EEG Signal Using Different Filters", International Journal of Innovative Research and Development, 2(4):915-927, 2013

[8]. Daqrouq, K.; Ajour, M.; Al-Qawasmi, A.R.; Alkhateeb, A. "The discrete wavelet transform based electrocardiographic baseline wander reduction method for better signal diagnosis",. J. Med. Imag. Health Inf.2018, 8, 1590-1597.

[9]. Lee, J.S.; Heo, J.; Lee, W.K.; Lim, Y.G.; Kim, Y.H.; Park, K.S. "Flexible capacitive electrodes for minimizing motion artifacts in ambulatory electroencephalogram s". Sensors 2014, 14, 14732-14743.

[10]. Yin, C.; Zhou, H.; Li, J. Facile "one-step hydrothermal synthesis of PEDOT: PSS/MnO2 
nanorod hybrids for high-rate supercapacitor electrode materials",. Ionics 2019, 25, 685695.

[11]. Michael R Gold et al. "The effect of TDAB duration and morphology on cardiac resynchronization therapy outcomes in mild heart failure: results from the resynchronization reverses Remodeling in Systolic left ventricular dysfunction (REVERSE) Study", Circulation, page 112, 2012.

[12]. Mei, Y.; Tan, G.Z.; Liu, Z.T.; Wu, H. “Chaotic time series prediction based on brain emotional learning model and self-adaptive genetic algorithm”. Acta Phys. Sin. 2018

[13]. He, S.; Sun, K.; Wang, R. "Fractional fuzzy entropy algorithm and the complexity analysis for nonlinear time series". Eur. Phys. J.-Spec. Top. 2018, 227, 943-957

[14]. Hashim, F.R.; Adnan, J.; Daud, N.G.N.; Mokhtar, A.S.N.; Rashidi, A.F.; Rizman, Z.I., "Electroencephalogram noise cancellation using wavelet transform.", J. Fundam. Appl. Sci. 2017, 9, 131-140

[15]. Feng, D.S.; Yang, D.X.; Wang, X. "Ground penetrating radar numerical simulation with interpolating wavelet scales method and research on fourth-order Runge-Kutta auxiliary differential equation perfectly matched layer", Acta Phys. Sin. 2016, 65, 23

[16]. Octaviani, V.; Kurniawan, A.; Suprapto, Y.K.; Zaini, A. "Alerting system for sport activity based on EEG signals using proportional integral derivative", In Proceedings of the 2017 4th International Conference on Electrical Engineering, Computer Science and Informatics (EECSI), Yogyakarta, Indonesia, 19-21 September 2017; pp. 1-6

[17]. Mamaghanian.H, Khaled.N, Atienza D, Vandergheynst P. "Compressed sensing for real-time energy-efficient EEG compression on wireless body sensor nodes", IEEE Trans. Biomed. Eng. 2011, 58, 2456-2466.

[18]. Karnewar, J.S.; Shandilya, D.V.K.; Tambakhe, M.D. "A study on EEG signal analysis and EEG databases”. Int. J. Res. Advent Technol. 2019, 7, 188-19

[19]. Tychkov, A.; Alimuradov, A.; Churakov, P. "The emperical mode decomposition for EEG signal preprocessing". In Proceedings of the 2019 3rd School on Dynamics of Complex Networks and their Application in Intellectual Robotics, DCNAIR”, Innopolis, Russia, 9-11 December 2019
[20]. K. Kalimeri and C. Saitis, "Exploring multimodal biosignal features for stress detection during indoor mobility," in Proceedings of the 18th ACM International Conference on Multimodal Interaction, ICMI 2016, pp. 53-60, Japan, November 2016.

[21]. Foud Amin and Mohamed mostafafouad, "Brain-Computer Interface: A Review", 2015.

[22]. J. R.Wolpaw, N. Birbaumer, D. J. McFarland, G. Pfurtscheller, and T. M. Vaughan, "Braincomputer interfaces for communication and control." ClinNeurophysiol, vol. 113, pp. 767791, Jun. 2002.

[23]. A. Kubler, F. Nijboer, J. Mellinger, T. M. Vaughan, H. Pawelzik, G. Schalk, D. J. McFarland, N. Birbaumer, and J. R. Wolpaw, "Patients with ALS can use sensorimotor rhythms to operate a brain-computer interface." Neurology, vol. 64, no. 10, pp. 1775-7, May 2005.

[24]. Ian Daly, Reinhold Scherer, Martin Billinger, and GernotM"ullerPutz, "FORCe for brain computer interface" 2015

[25]. Sebastian Halder and Wolfgang Rosenstiel, "online artifact removal for Brain-Computer Interface using support vector machine" IEEE Aug 2007.

[26]. Luis Fernando Nicolas-Alonso and "BrainComputer Interfacing” Jan 2012.

[27]. H. Nolan, R. Whelan, and R. B. Reilly, "FASTER: Fully Automated Statistical Thresholding for EEG artifact Rejection." Journal of neuroscience methods, vol. 192, no. 1, pp. 152-62, Sep. 2010.

[28]. I. Daly, N. Nicolaou, S. J. Nasuto, and K. Warwick, "Automated artifact removal from the electroencephalogram: a comparative study." Clinical EEG and neuroscience, vol. 44, no. 4, pp. 291306 , Oct.2013.

[29]. P. S. Kumar, R. Arumuganathan, K. Sivakuma, and C. Vimal, "Removal of ocular artifacts in the EEG through wavelet transform without using an EOG reference channel," vol. 1, no. 3, pp. 188-200, 2002.

[30]. Veer AmolMotinath, Chandan Kumar and Maheshkumar H Kolekar "A Novel ECG Data Compression Algorithm using Best Mother Wavelet Selection", Sept 2016.

\section{Creative Commons Attribution License 4.0 (Attribution 4.0 International, CC BY 4.0)}

This article is published under the terms of the Creative Commons Attribution License 4.0

https://creativecommons.org/licenses/by/4.0/deed.en_US 